\title{
Orientational Disorder in Epitaxially Connected Quantum Dot Solids
}

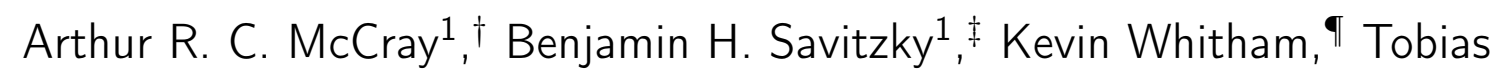
Hanrath, ${ }^{\S}$ and Lena F. Kourkoutis*,,,$\|$

$\dagger$ School of Applied and Engineering Physics, Cornell University, Ithaca, NY 14853, USA

$\ddagger$ Department of Physics, Cornell University, Ithaca, NY 14853, USA

IDepartment of Materials Science and Engineering, Cornell University, Ithaca, NY 14853, USA

§School of Chemical and Biomolecular Engineering, Cornell University, Ithaca, NY 14853, USA

||Kavli Institute for Nanoscale Science, Cornell University, Ithaca, NY 14853, USA

E-mail: lena.f.kourkoutis@cornell.edu

${ }^{1}$ A. McCray and B. Savitzky contributed equally to this work. 


\section{All QD Orientations and Sizes}

Figure 1 shows a histogram of average radius of the 1823 QDs visible across 4 images. As the QDs are not perfectly round, average radius was defined as the radius of a circle with the same area as the QD. The mean radius across all QDs was found to be $6.7 \mathrm{~nm}$ with a $0.4 \mathrm{~nm}$ standard deviation.

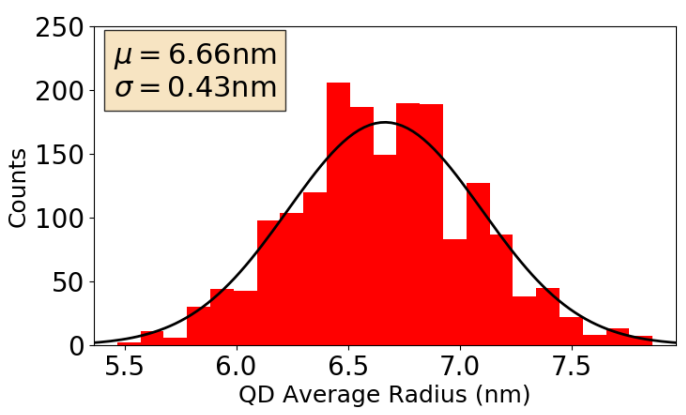

Figure 1: Size distribution of 1823 QDs. Average radius is defined by a circle corresponding to the QD area.

Of the 1823 QDs, 95.1\% were oriented such that some form of atomic lattice periodicity was identifiable. We segmented the images, masked individual QDs with a Hann window to remove edge effects, and took FFTs of each QD. By locating Bragg peaks, we were able to reliably extract spatial frequency and symmetry information from the AL for each QD. All QDs with visible lattices were found to be in one of five orientations, shown in Fig. S1. Two of these were oriented directly along crystallographic axes, the $\langle 100\rangle$ and $\langle 110\rangle$. The other three orientations were partially tilted, such that lattice periodicity was only identifiable in one direction. QDs with intermediate out-of-plane orientations between the $\langle 100\rangle$ and $\langle 110\rangle$ were observed, and are here referred to as $\langle 1 x 0\rangle$. We believe (see main text and Fig. S6) that $|x| \ll 1$, i.e. the mistilt from the $\langle 100\rangle$ direction is small. Similarly, we find QDs oriented between the $\langle 100\rangle$ and $\langle 111\rangle$ directions which we refer to as $\langle 1 y y\rangle$. We observe a small fraction of QDs with out-of-plane orientations in between the $\langle 110\rangle$ and $\langle 111\rangle$ orientations, which we call $\langle 1(z+1) z\rangle$. 


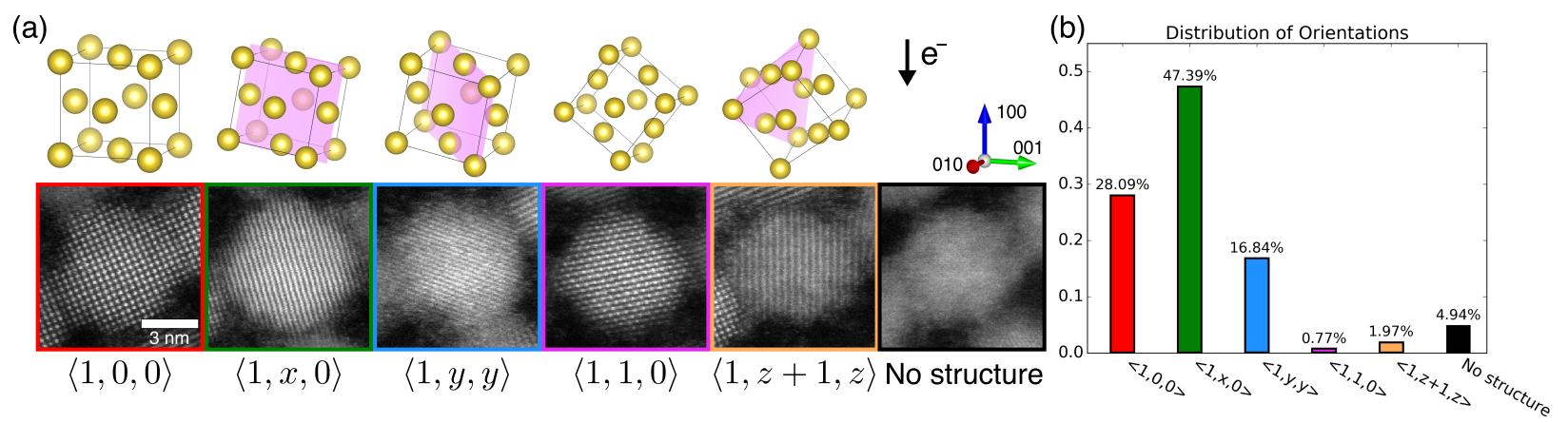

Figure 2: Out-of-plane orientations of the QD ALs. (a) We observed five distinguishable orientations. Two of these categories had crystallographic axes, the $\langle 100\rangle$ and $\langle 110\rangle$ directions, aligned out-of-plane. The remaining three were partially rotated out-of plane. Rotation planes for off-axis QDs are represented in pink, and corresponding STEM images are below. (b) Distribution of the 1823 QDs across the orientations. 


\section{Determining Nearest Neighbors}

When comparing AL orientations between QDs, we only want to look at neighbors that are epitaxially connected or centered close enough together such that they could be connected. Savitzky et al. examined distances and epitaxial connections between neighboring QDs. ${ }^{1}$ Following this work, we selected $7.4 \mathrm{~nm}$ as the cutoff distance used to define near neighbors. QDs centered less than $7.4 \mathrm{~nm}$ apart were close enough to be connected QDs, regardless of whether the epitaxial bonds were actually formed, and therefore their corresponding AL orientations were deemed relevant.

Using this metric, Fig. 3 shows the average number of neighbors in blue for all 1823 QDs examined. Somewhat surprisingly, the average is approximately three close neighbors fpr QD. We observe nominally more neighbors for $\langle 100\rangle$ QDs than $\langle 1 x 0\rangle$ and $\langle 1 y y\rangle$, and that trend is strengthened when just counting neighbors that are oriented close to $\langle 100\rangle$ (i.e. $\langle 100\rangle,\langle 1 x 0\rangle$, or $\langle 1 y y\rangle$ as shown in green). Poorly aligned QDs that are further from $\langle 100\rangle$ (and QDs for which we could not at all determine their orientation) are less likely to be close to well-aligned QDs as shown by the increasing gap between the two averages.

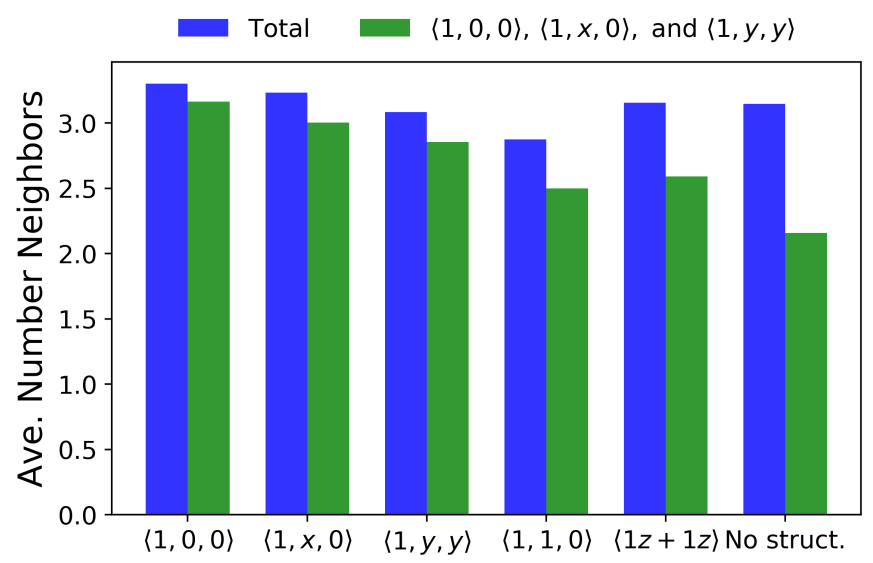

Figure 3: The average number neighbors for each QD orientation using a $7.4 \mathrm{~nm}$ cutoff radius. Blue considers all QDs, green counts only neighbors that are $\langle 100\rangle,\langle 1 x 0\rangle$, or $\langle 1 y y\rangle$.

Knowing the average number of neighbors per QD also helps us learn about the relationship between QD orientations and their neighbors. By recreating the histograms of Fig. 1g 
with $\alpha_{i}$ instead of $\alpha_{n}$, we find the the standard deviations increase by very nearly $\sqrt{3}$, which is consistent with the hypothesis that $\alpha_{i}$ are uncorrelated. The wider spread of these histograms is due to highly misaligned individual neighbors which are brought closer to the norm when averaged for $\alpha_{n}$.

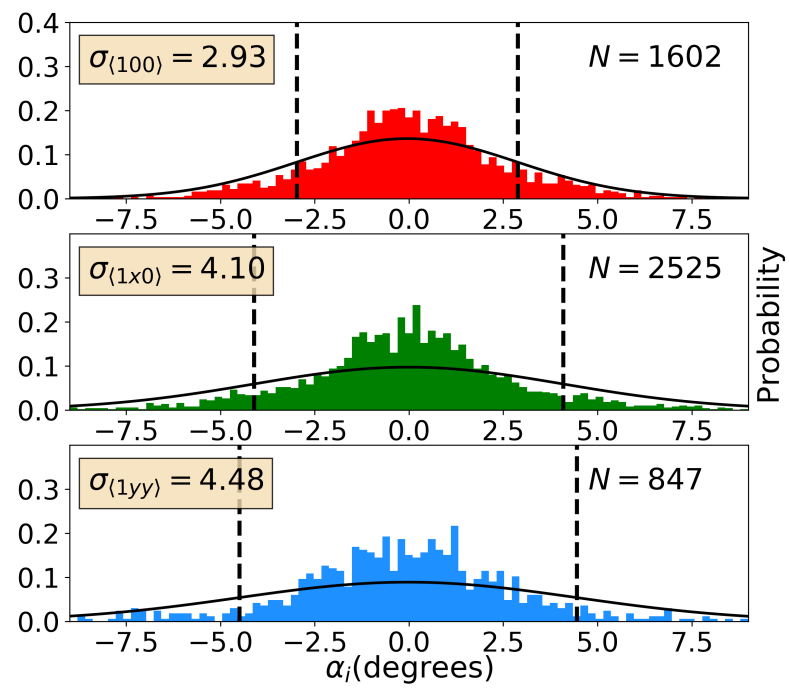

Figure 4: Histograms for misalignments from all individual neighbors for QDs in $\langle 100\rangle$ (red), $\langle 1 x 0\rangle$ (green), or $\langle 1 y y\rangle$ (blue) orientations. The standard deviations are close to $\sqrt{3}$ the value when plotting $\alpha_{n}$, as one would expect for uncorrelated $a l p h a_{i}$ and an average of 3 neighbors per QD.

For the purpose of defining the local superlattice metric $\psi_{4}$, looking at only bonded neighbors would give an inaccurately high measure of symmetry for QDs in disordered regions. For instance, it would be possible for a QD in a very disordered region to have only one or two epitaxially connected neighbors, with additional highly sixfold symmetric QDs slightly further away. To take this into account, when calculating $\psi_{4}$ we define a particular QD's neighbors as all QDs with which it shares a Voronoi cell edge. This is a choice commonly used when dealing with colloidal or hard packing nanoparticles ${ }^{2-6}$ as it unambiguously defines neighbors even in chaotic regions. Additionally, the Voronoi diagram enables weighting the importance of each neighbor using the length of the shared edge. 


\section{Error in Segmentation and $\psi_{4}$}

The segmentation process finding QD centroids is critical in this analysis as it directly provides the quantification of local SL symmetry through $\psi_{4}$, as well AL information after FFTs are taken of individual QDs. Figure 5 shows an overview of the four main steps of segmentation. We begin by thresholding using a local minimum value in the intensity profile of the image; this makes a rough mask. A number of morphological openings and closings are performed to clean the image and a distance transformation is applied. Local maxima can be detected, and these are used as sources in a watershed algorithm that segments the image within the confines of the mask. Centroids are then determined using the center-of-mass for each QD.

Error can occur in each of these steps, with a non-optimal threshold value the most damaging. If too small a value is chosen, many QDs blur together and accuracy decreases, while if the threshold is too large, entire QDs are lost. Errors can also be introduced if the thresholded image isn't cleaned enough or if incorrect peaks are identified after the distance transform.

Figure 6 shows the quantitative results of these errors. Full segmentation is run on one image for varying parameters: alternate threshold values of 1.06 and 0.9 times the optimal value used (blue and yellow), half the morphological transformations (green), and poor peak detection (pink). Fewer morphological transformations creates a grainy mask, and failing to select for weaker intensity peaks means small QDs are missed.

The QD centroids for these changes as well as the optimized parameters are plotted in Fig. 6a; for most QDs, all centers fall within a few pixels of each other. Figure 6b shows the RMS values of each alternate segmentation compared to the optimized centroids. It is clear that thresholding has the largest effect, but in all cases error is less than $0.13 \mathrm{~nm}$. Likewise for $\left|\psi_{4}\right|$ and $\phi_{4}$, the error is on the order of 0.025 and 0.4 degrees. These values are much smaller than the measured variation which gives weight to our conclusions and using $\psi_{4}$ as an order metric. 


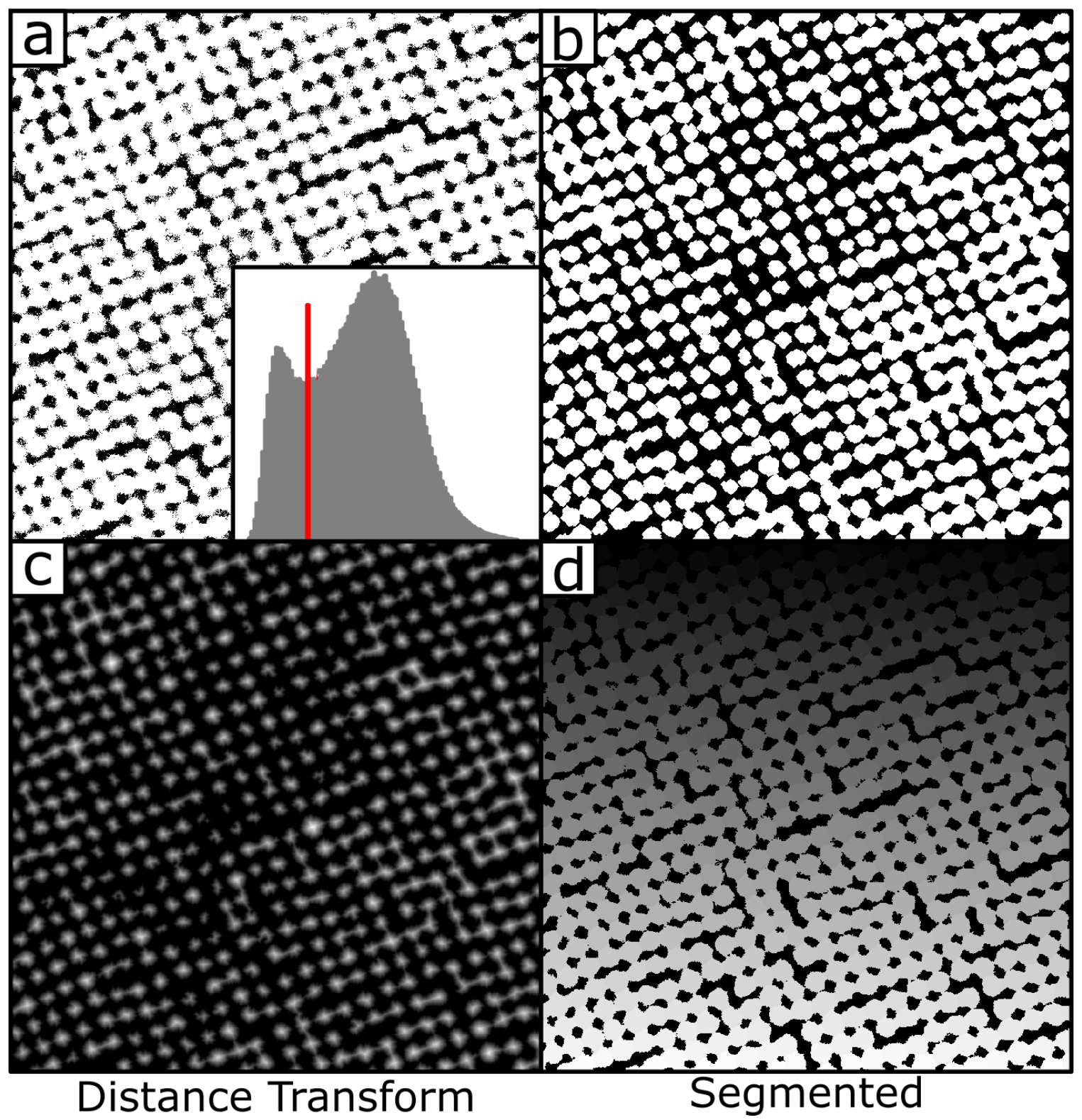

Figure 5: The primary steps in the segmentation process. (a) The original image is thresholded using a hand-chosen value picked to be the local minima of the image's intensity histogram (inset). (b) It is then cleaned using a variety of morphological transformations. (c) A distance transform is applied and local maxima are identified as approximate centers. (d) These are used as sources in a watershed algorithm to get a segmented image. 


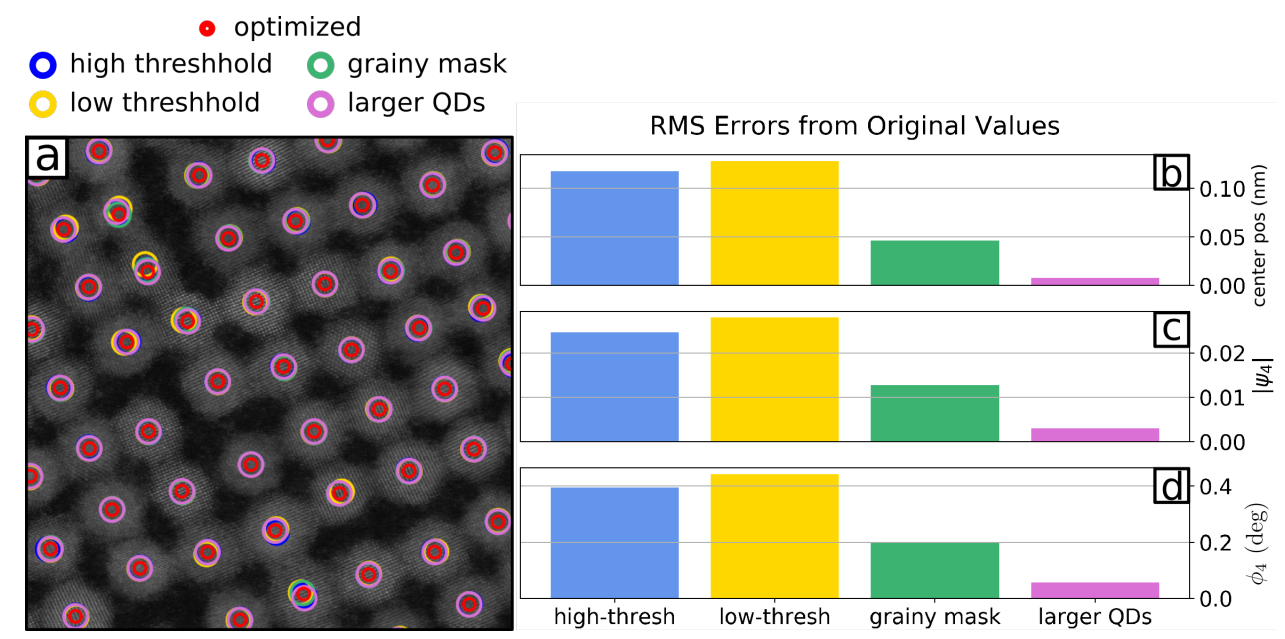

Figure 6: Error in segmentation and $\psi_{4}$. (a) The segmented QD centers overlaid for our optimized algorithm (red) and with various parameters changed. Blue: threshold too large. Yellow: threshold too small. Green: not enough mask cleaning. Pink: too large of QDs expected. Right: RMS errors calculated against the optimized segmentation for all QDs in the image. Histograms show for each changed parameter the final error in (b) centroid position, (c) $\left|\psi_{4}\right|$, and (d) $\phi_{4}$. 


\section{Remaining Sixfold Order}

We observe a rough spatial anti-correlation between $\left|\psi_{4}\right|$ and $\left|\psi_{6}\right|$. Parts of the SL with low four-fold order tend to be higher in six-fold symmetry. This is likely a result of remaining six-fold symmetry from the close-packing arrangement of the QDs prior to the oriented attachment process. We also observe some regions with $1 \mathrm{D}$ strips of good fourfold order in the SL.

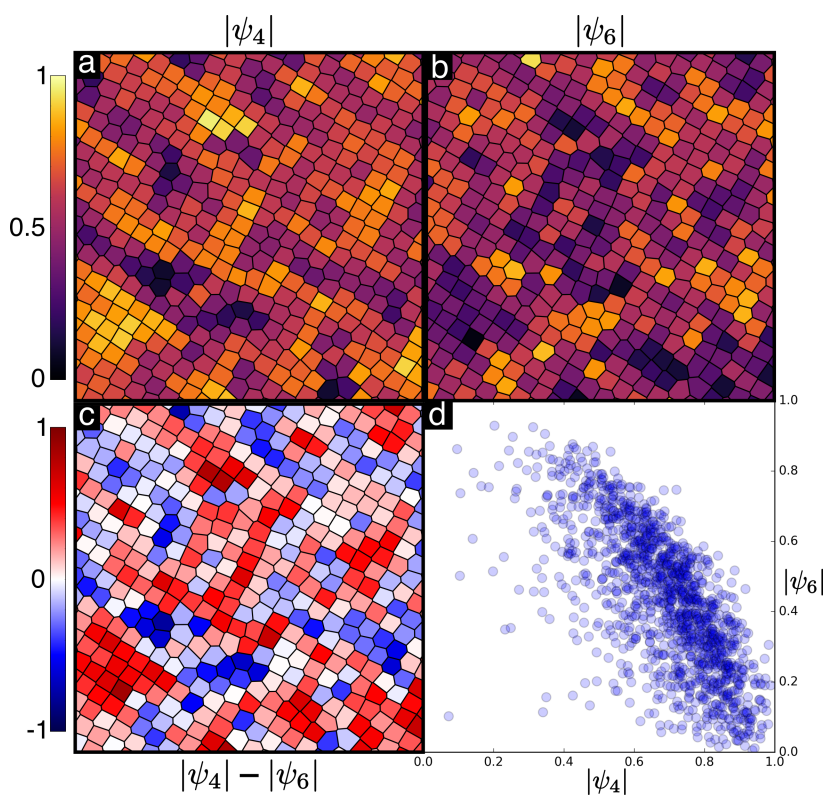

Figure 7: QDs with low $\left|\psi_{4}\right|$ tend to have higher $\left|\psi_{6}\right|$. (a) The local magnitude of fourfold symmetry, $\left|\psi_{4}\right|$. Regions with high fourfold symmetry often occur in 1D strips of QDs. (b) The local magnitude of sixfold symmetry, $\left|\psi_{6}\right|$. Sixfold symmetry appears roughly spatially anti-correlated with fourfold order. (c) The difference between $\left|\psi_{4}\right|$ and $\left|\psi_{6}\right|$. (d) Plotting $\left|\psi_{4}\right|$ against $\left|\psi_{6}\right|$ confirms their anti-correlation. 


\section{Localized Neck Bending}

Bending of the AL within the adjoining neck shown in Fig. 4 of the main text occurs essentially entirely within 3 unit cells, as shown in Fig. 8, suggesting the significant energetic cost of misalignment between connected QDs. The spatial extent of the bent region was determined using Fourier analysis following Hytch's geometric phase analysis (GPA) approach. ${ }^{7,8}$ Our simple approach to determining the localization of the neck bending via GPA is briefly described below.

In GPA, the image of an imperfect lattice is taken to be described by

$$
I(\mathbf{r})=\sum_{\mathbf{g}} c_{\mathbf{g}}(\mathbf{r}) e^{2 \pi i \mathbf{g} \cdot \mathbf{r}}
$$

for image intensity $I(\mathbf{r})$ at real space coordinate $\mathbf{r}$, and reciprocal lattice vectors $\mathbf{g}$. For a perfect lattice, the $c_{\mathbf{g}}(\mathbf{r})=c_{\mathbf{g}}$, i.e., are constant, and Eq. 1 reduces to a Fourier series expansion. Any deviation from a perfect lattice is therefore captured here by the spatial dependence of the $c_{\mathbf{g}}(\mathbf{r})$. The Fourier transform of Eq. 1 gives

$$
\tilde{I}(\mathbf{k})=\sum_{\mathbf{g}} \tilde{c}_{\mathbf{g}}(\mathbf{k}-\mathbf{g})
$$

where the tildes indicate a Fourier transform. Algorithmically, then, for some value of $\mathbf{g}$ the spatially dependent Fourier coefficient $c_{\mathbf{g}}(\mathbf{r})$ can be extracted by taking the Fourier transform of the image, shifting the origin of the output to $\mathbf{g}$, discarding information outside the first Brillouin zone (i.e. selecting only the $\mathbf{g}$ of interest), and inverse Fourier transforming. Using a mask smaller than the first Brillouin zone to discard information from other reciprocal lattice vectors can help improve SNR, at the cost of reduced spatial resolution.

Once $c_{\mathbf{g}}(\mathbf{r})$ is known, it can be used to identify strain or other variations in the lattice. 
$c_{\mathbf{g}}(\mathbf{r})$ is complex valued and may be written as

$$
c_{\mathbf{g}}(\mathbf{r})=A_{\mathbf{g}}(r) e^{i P_{\mathbf{g}}(\mathbf{r})}
$$

for real valued functions $A_{\mathbf{g}}(\mathbf{r})$ and $P_{\mathbf{g}}(\mathbf{r})$. The object of interest is typically the phase, $P_{\mathbf{g}}(\mathbf{r}) . A_{\mathbf{g}}(\mathbf{r})$ describes the strength of the periodicity under study at $\mathbf{r}$. Local variations in the reciprocal lattice vector $\Delta \mathbf{g}$ can be calculated from the gradient of the phase

$$
\nabla P_{\mathbf{g}}(\mathbf{r})=2 \pi \Delta \mathbf{g}
$$

The selected $\mathbf{g}$ is indicated by the red circle in the inset of Fig. 8a. To isolate the regions of interest, Fig. 8b shows the phase times a binary filter in the amplitude, i.e.

$$
P_{\mathbf{g}}(\mathbf{r}) \Theta\left(A_{\mathbf{g}}(\mathbf{r})-A_{0}\right)
$$

where $\Theta$ is the Heaviside function and $A_{0}$ is some threshold constant. As we are interested in the bending of the lattice, rather than compressive/tensile strain, the relevant components of Eq. 4 are those perpendicular to g, i.e.

$$
\frac{\partial}{\partial r_{\perp}} P_{\mathbf{g}}(\mathbf{r})=2 \pi \Delta g_{\perp}
$$

Figure $8 c$ shows a line profile of the phase along the arrows shown in (a) and (b), which are approximately perpendicular to the selected $\mathbf{g}$. The slope of the line profile gives $\frac{\partial}{\partial r_{\perp}} P_{\mathbf{g}}(\mathbf{r})$. The regions on the left and right with approximately constant slopes indicate no bending, and to the bulk of the left and right QDs. The difference between the two constant slopes gives the rotation between the QDs, discussed above, via $\tan \theta=\frac{\Delta g_{\perp}}{|\mathbf{g}|}$. Finally, essentially all of the lattice bending occurs in the region between the two red arrows where $\frac{\partial}{\partial r_{\perp}} P_{\mathbf{g}}(\mathbf{r})$ is non-constant. The bending is localized to region of no more than $\sim 1.5 \mathrm{~nm}$, or $2.5 \mathrm{PbSe}$ 
unit cells. Note that a mask smaller than the first Brillouin zone was used before inverse Fourier transforming, reducing the resolution of this estimate, thus this quantity should be considered an upper bound on the spatial extent of the lattice bending.
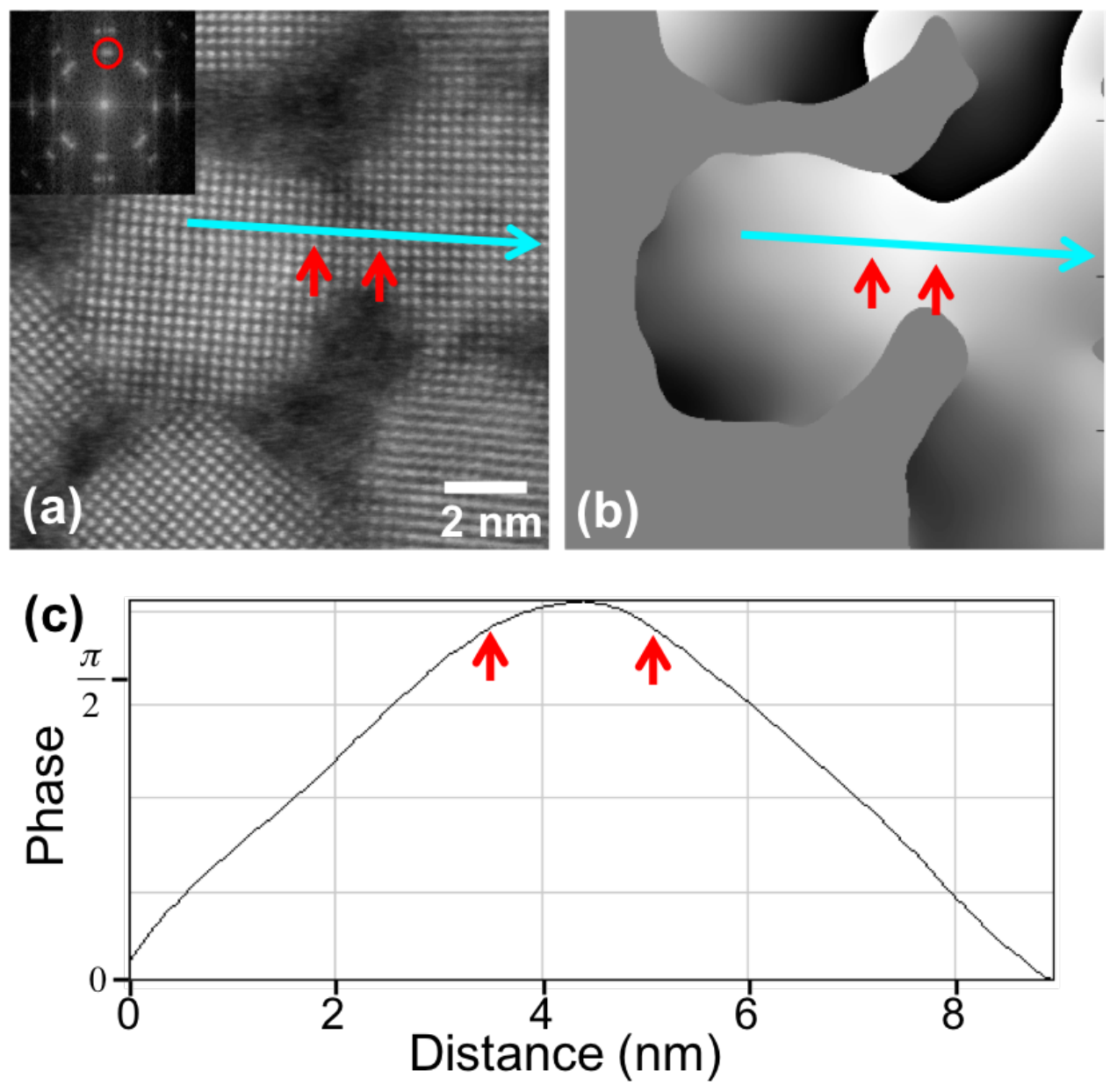

Figure 8: Localization of strain in bent epitaxial necks. (a) The STEM image from Fig. 4 of the main text. The red circle in the inlaid FFT shows the Fourier peak used for GPA. (b) The masked phase map (Eq. 5) corresponding to the phase of the two attached QDs of interest and their connecting bridge. (c) A line profile of the phase map (b) across the teal line indicated in $(a, b)$. Essentially all of the atomic lattice bending strain occurs within the region between the two red arrows where the phase is non-constant, spanning a width of $\sim 2.5$ unit cells. 


\section{Epitaxial Bridges}

The epitaxial connections that enhance charge transport between QDs should promote alignment between ALs. We find that while there are many examples of bridges between QDs in the same orientation (see Fig. 9), they also occur between QDs misaligned in-plane by several degrees, as well as between QDs with different out-of-plane orientations. This indicates that ligand shells are not the cause of orientational misalignments.

This section provides a catalog of epitaxial bridges and their varieties.

Figure 9 shows âĂIJidealâĂİ epitaxial connections. Both QDs are aligned in the $\langle 100\rangle$ orientation, their atomic lattices are well aligned with one another, and the adjoining epitaxial bridges appear to be smooth continuations of the lattice in the QD cores. For bridges like these, the tunnel coupling is predicted to depend primarily on the bridge cross sections.

Figure 10 shows pairs of QDs which are both aligned âĂIJface upâĂ with the $\langle 100\rangle$ direction oriented out-of-plane, but which are misoriented relative to one another in the in-plane direction, such that the epitaxial bridges connecting the QDs are bent. Original images are shown above, while below the same images are shown overlaid with red lines along the directions of the ALs of the two particles.

Figures 11 and 12 show pairs of QDs which are misaligned in the out-of-plane direction. One QD in each pair is in the $\langle 100\rangle$ orientation. In Fig. 11 the second QD is in the $\langle 1 x 0\rangle$ orientation, and in Fig. 12 the second QD is in the $\langle 1 y y\rangle$ orientation. In spite of these misorientations, the epitaxial bridges connecting the QDs still display continuous, uninterrupted lattices, suggesting that the out-of-plane misorientation of the $\langle 1 x 0\rangle$ and $\langle 1 y y\rangle$ oriented QDs may be small.

Figure 13 shows epitaxial connections with defects in their ALs. Defects are apparant as reduced contrast at one or more atomic columns. The defect sites appear near the center of the bridges, suggesting they result from imperfect fusion of the two lattices during oriented attachment. Prior studies have observed that different outcomes may result from defects in the oriented attachment process, including quickly corrected defects through lat- 
tice restructuring, or a process of concomitant annihilation/growth as the mass of one QD is absorbed in the second via Ostwald ripening. ${ }^{9-11}$ Recent work examined the differing removal mechanisms for defects in bridges along different crystallographic axes. ${ }^{12}$ Here, we find stable defects at the center of the bridges. More detailed interpretation of defect types is challenging dues to the nature of projection imaging and electron channeling effects.

Figure 14 shows QDs with lattices in significantly different orientations, directly abutting one another. In these cases, the bridges do not appear to constitute a smooth, continuous AL connecting the two dots, but instead suggest the QDs are connected via $\sim$ nm scale grain boundaries.

Figure 15 shows very narrow bridges, with widths ranging from 1 to 5 atoms. We surmise that the mechanics of bridge bending and lattice deformation depends sensitively on the bridge width in the atomically fine limit.

Figure 16 shows epitaxial connections between QDs which occur along the [110], rather than the [100], facets. 

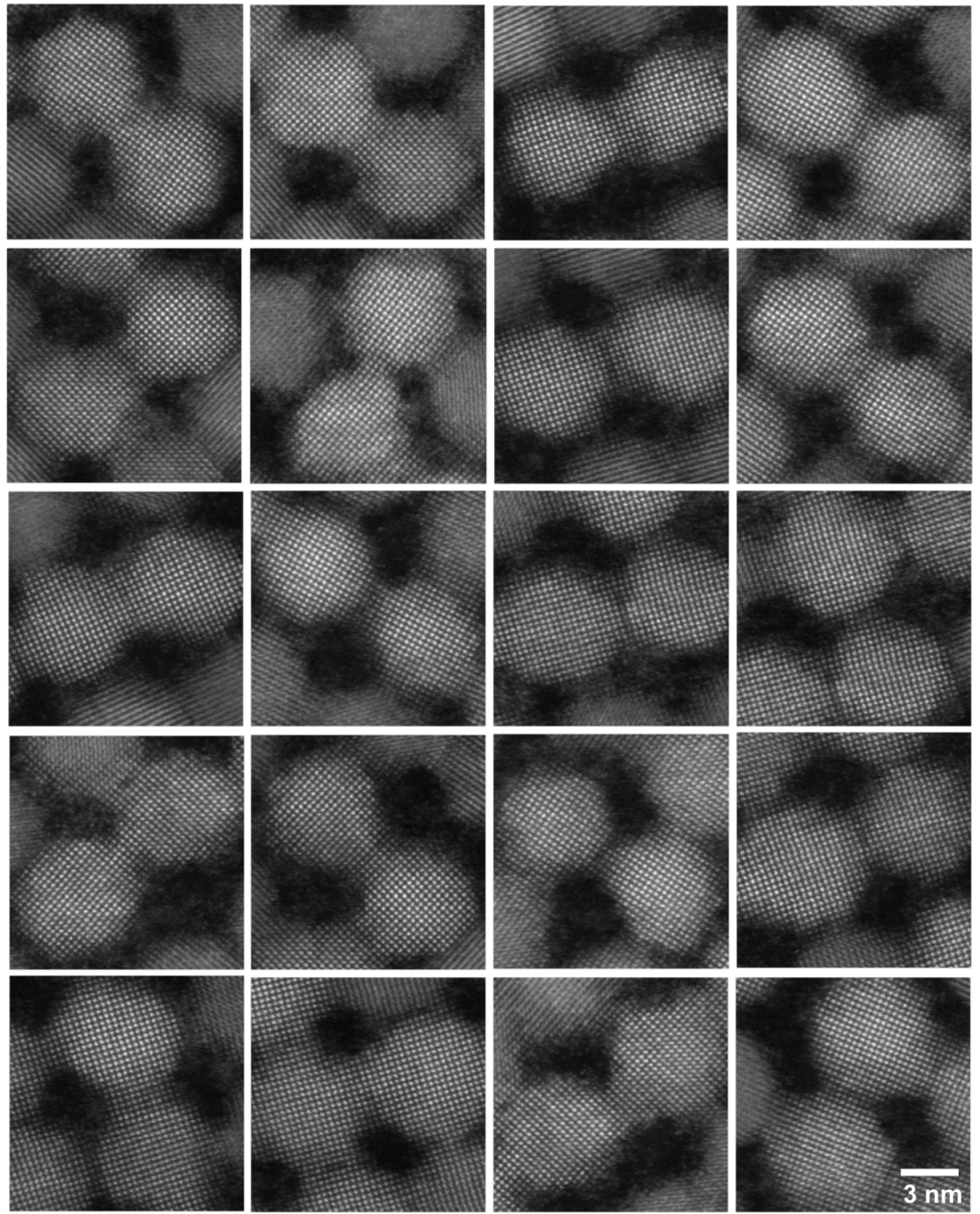

Figure 9: "Ideal" epitaxial connections between well aligned QDs oriented in the expected $\langle 100\rangle$ direction. 

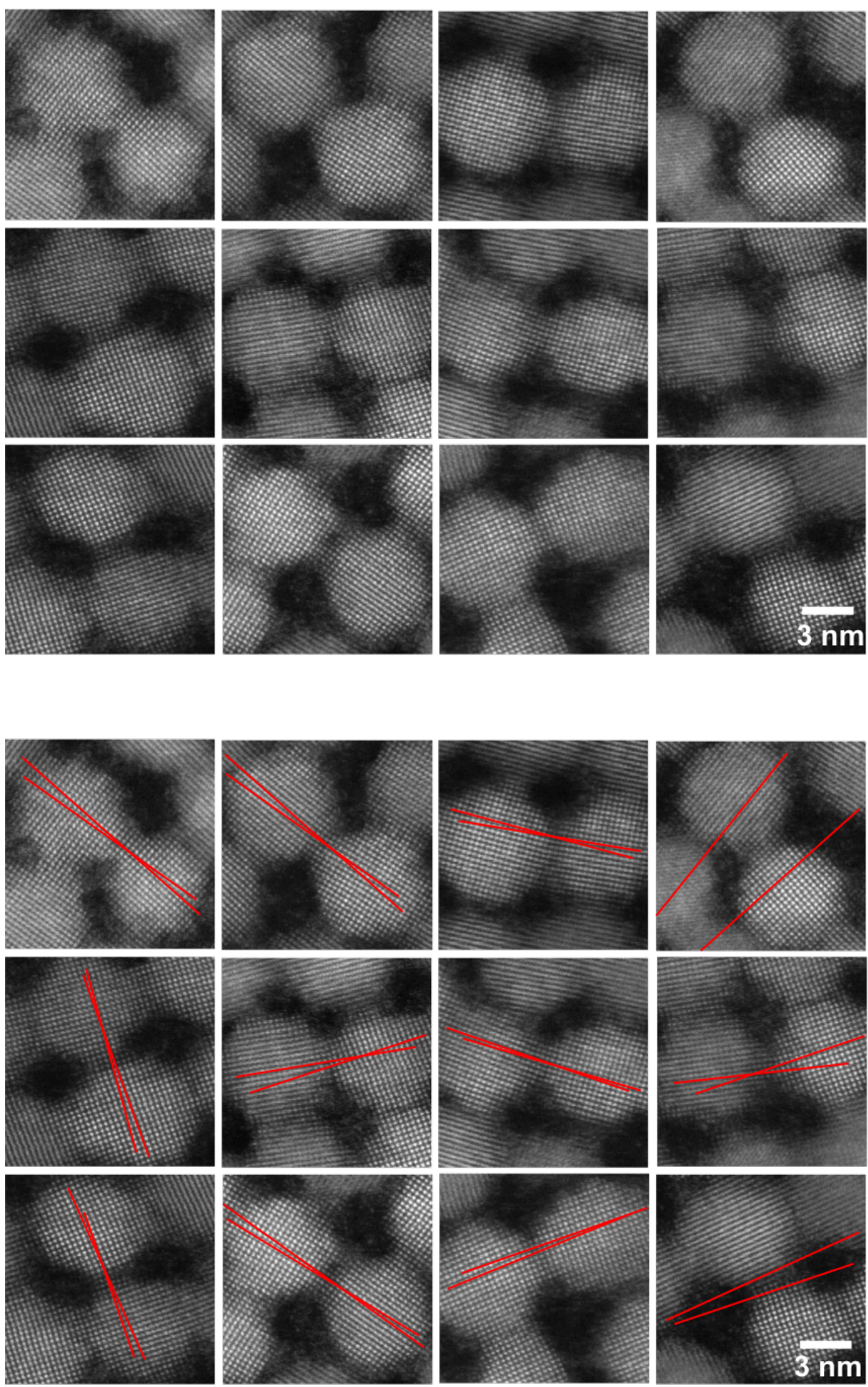

Figure 10: Bent bridges, in which the ALs of the connected QDs are misaligned in the inplane direction. Raw data is shown above, while below the red lines indicate the directions of the two QDsâĂŹ ALs in the same data. 

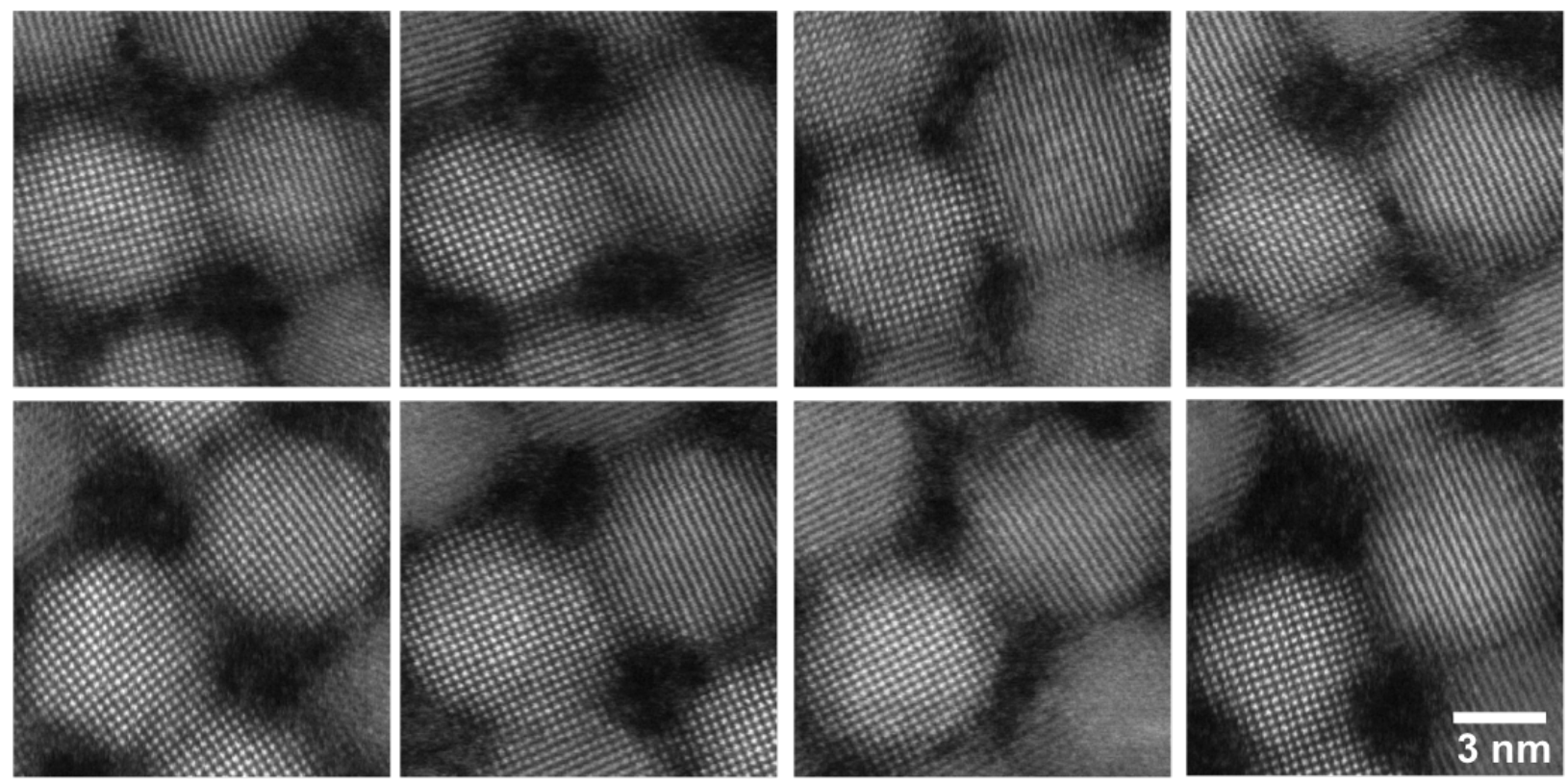

Figure 11: Tilted bridges, in which the ALs of the connected QDs are misaligned in the out-of-plane direction. Here, connections between $\langle 100\rangle$ and $\langle 1 x 0\rangle$ oriented QDs are shown, suggesting that the out-of-plane mistilt of the $\langle 1 x 0\rangle$ particles is small relative to the $\langle 100\rangle$ direction.
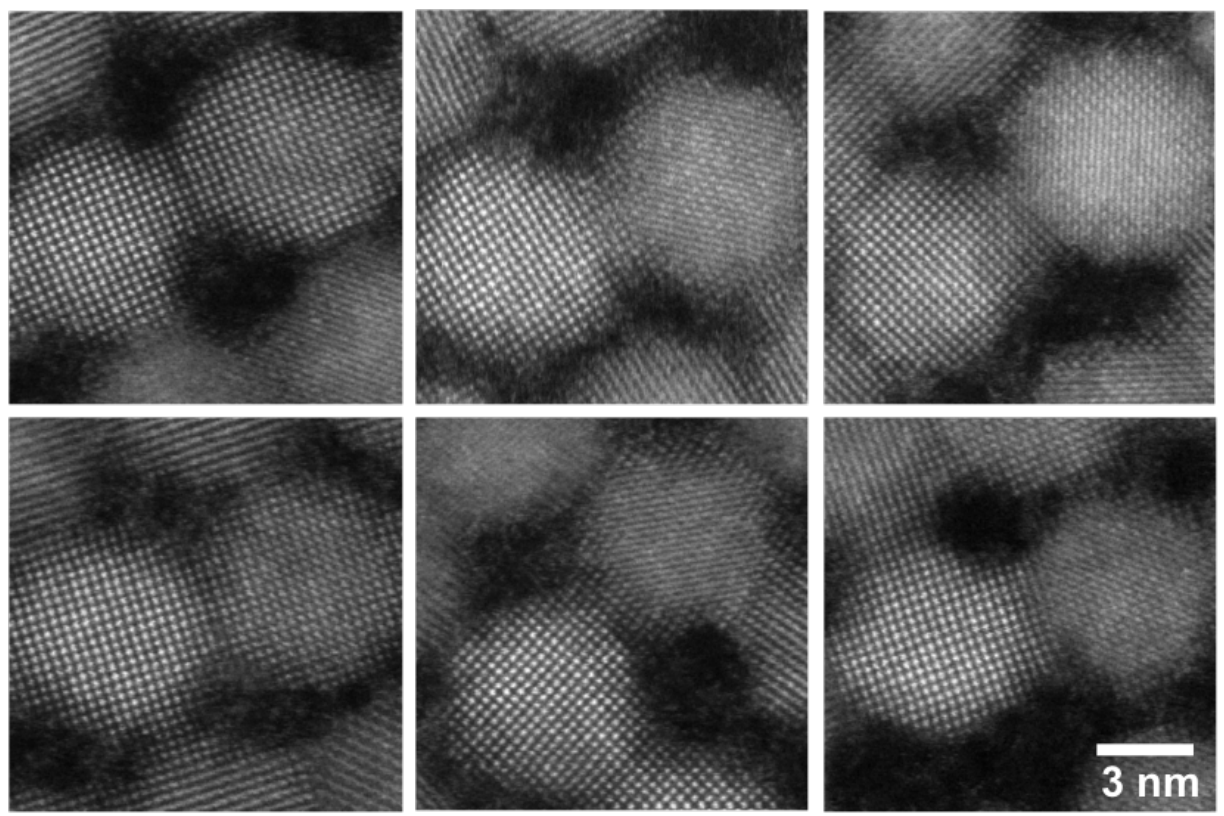

Figure 12: Tilted bridges, in which the ALs of the connected QDs are misaligned in the out-of-plane direction. Here, connections between $\langle 100\rangle$ and $\langle 1 y y\rangle$ oriented QDs are shown. 

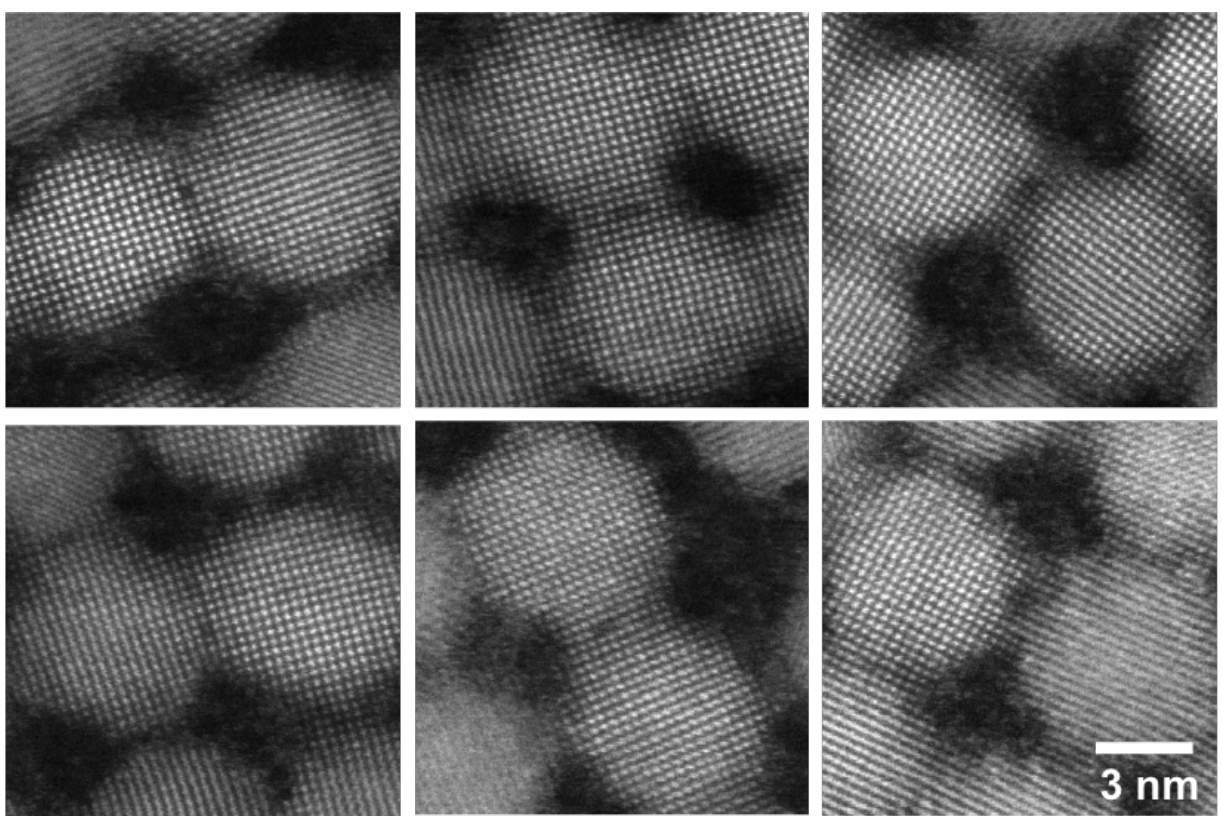

Figure 13: Defective bridges, with a lattice defect near the center of the neck.
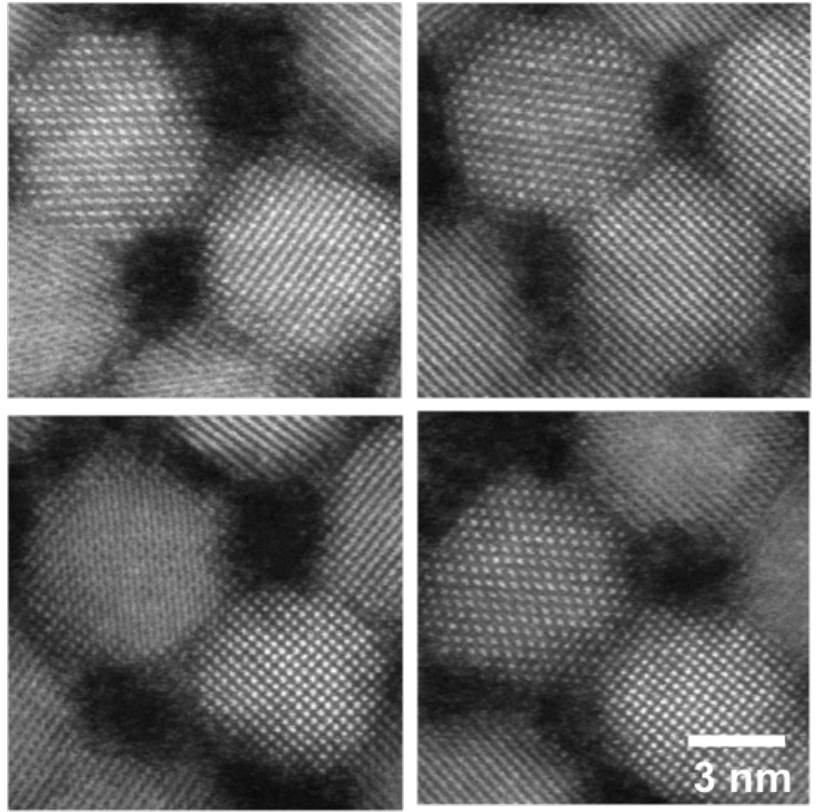

Figure 14: Non-continuous bridges, in which there is a discrete change in lattice orientations between the two abutting QDs, constituting minute grain boundaries. 

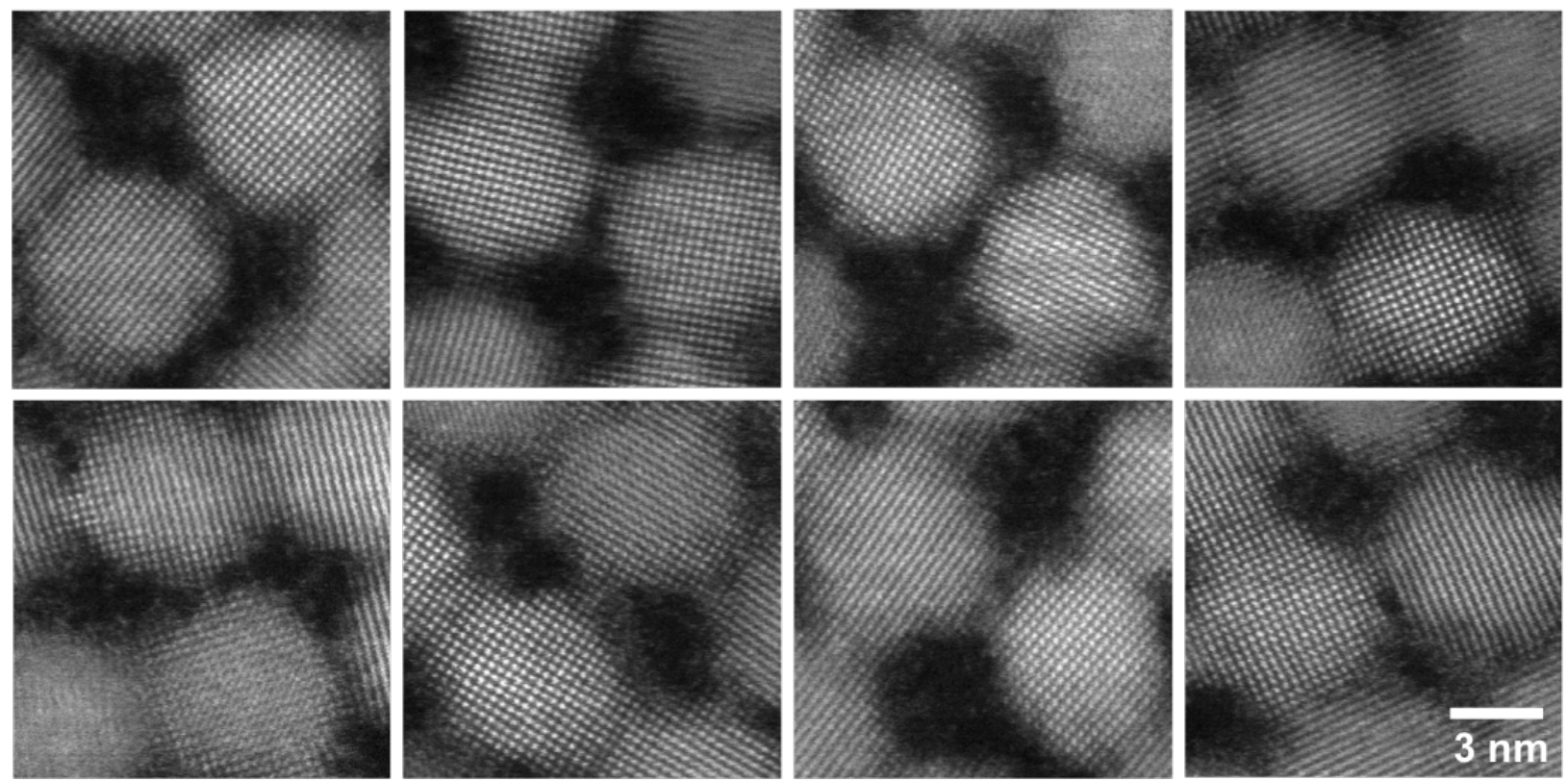

Figure 15: Very narrow bridges, with widths as small as a single atom, and up to five atoms across.
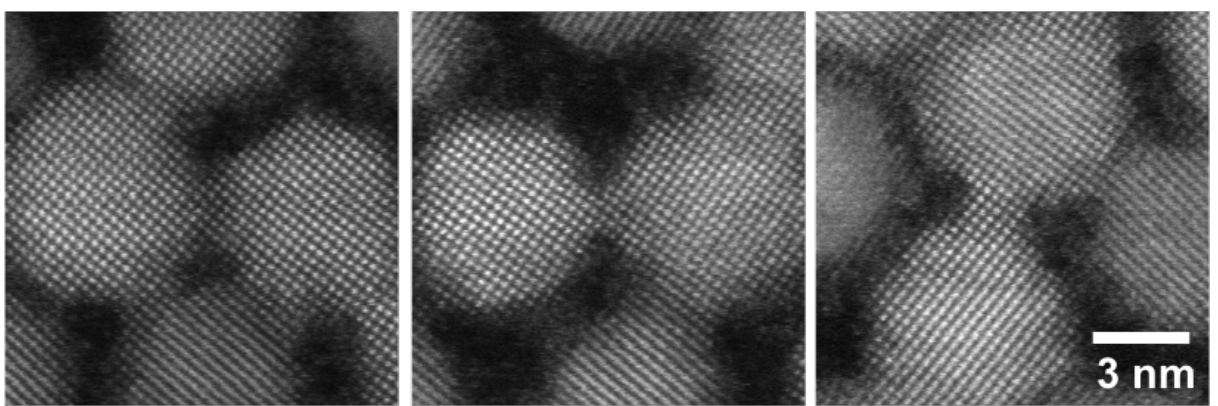

Figure 16: Bridges connecting [110] facets of adjacent QDs. 


\section{References}

1. Savitzky, B. H.; Hovden, R.; Whitham, K.; Yang, J.; Wise, F.; Hanrath, T.; Kourkoutis, L. F. Propagation of Structural Disorder in Epitaxially Connected Quantum Dot Solids From Atomic to Micron Scale. Nano Lett. 2016, 16, 5714-5718.

2. Mickel, W.; Kapfer, S. C.; Schröder-Turk, G. E.; Mecke, K. Shortcomings of the Bond Orientational Order Parameters for the Analysis of Disordered Particulate Matter. J. Chem. Phys. 2013, 138.

3. Steinhardt, P. J.; Nelson, D. R.; Ronchetti, M. Bond-Orientational Order in Liquids and Glasses. Phys. Rev. B 1983, 28, 784-805.

4. Lochmann, K.; Anikeenko, A.; Elsner, A.; Medvedev, N.; Stoyan, D. Statistical Verification of Crystallization in Hard Sphere Packings Under Densification. Eur. Phys. J. B 2006, 53, 67-76.

5. Xu, W. S.; Sun, Z. Y.; An, L. J. Dense Packing in the Monodisperse Hard-Sphere System: A Numerical Study. Eur. Phys. J. E 2010, 31, 377-382.

6. Calvo, F.; Mottet, C. Order-Disorder Transition in Co-Pt Nanoparticles: Coexistence, Transition States, and Finite-Size Effects. Phys. Rev. B 2011, 84, 19-21.

7. Hÿtch, M.; Snoeck, E.; Kilaas, R. Quantitative Measurement of Displacement and Strain Fields From HREM Micrographs. Ultramicroscopy 1998, 74, 131-146.

8. Hÿtch, M. J.; Putaux, J. L.; Pénisson, J. M. Measurement of the Displacement Field of Dislocations to $0.03 \AA$ by Electron Microscopy. Nature 2003, 423, 270.

9. Penn, R. L. Imperfect Oriented Attachment: Dislocation Generation in Defect-Free Nanocrystals. Science 1998, 281, 969-971. 
10. Lu, W.; Gao, P.; Jian, W. B.; Wang, Z. L.; Fang, J. Perfect Orientation Ordered In-Situ One-Dimensional Self-Assembly of Mn-Doped PbSe Nanocrystals. J. Am. Chem. Soc. 2004, 126, 14816-14821.

11. Li, D.; Nielsen, M. H.; Lee, J. R.; Frandsen, C.; Banfield, J. F.; De Yoreo, J. J. DirectionSpecific Interactions Control Crystal Growth by Oriented Attachment. Science 2012, 336, 1014-1018.

12. Ondry, J. C.; Hauwiller, M. R.; Alivisatos, A. P. Dynamics and Removal Pathway of Edge Dislocations in Imperfectly Attached PbTe Nanocrystal Pairs; Towards Design Rules for Oriented Attachment. ACS Nano 2018, 3178-3189. 